\title{
ТРАНСФОРМАЦИЯ ГЛОБАЛЬНЫХ ЦЕПЕЙ ПОСТАВОК В УСЛОВИЯХ РАЗВИТИЯ РЫНКА АДДИТИВНЫХ ТЕХНОЛОГИЙ
}

\author{
(C) 2018 Го Хунли \\ кафедра логистики и управления цепями поставок \\ Санкт-Петербургский государственный экономический университет \\ Россия, 191023, Санкт-Петербург, ул. Садовая, д. 21 \\ E-mail:go.hunli@yandex.ru \\ (c) 2018 Ценина Татьяна Тихоновна \\ кафедра логистики и управления цепями поставок \\ Санкт-Петербургский государственный экономический университет \\ Россия, 191023, Санкт-Петербург, ул. Садовая, д. 21 \\ E-mail: cakie@yandex.ru
}

Переход к новому технологическому укладу влечет за собой значительные трансформации в структуре промышленного производства и потребления. Одним из основных драйверов данных изменений выступают аддитивные технологии. Развитие рынка данных технологий влечет за собой трансформацию не только технологических процессов, но и сопутствующих им логистических процессов. Следствием данных трансформаций на макроуровне может стать не только содержательное, но и структурное преобразование глобальных цепей поставок. В рамках данной статьи авторами раскрывается специфика данных преобразований и рассматриваются принципиально новые модели цепей поставок, появляющиеся в условиях развития аддитивных технологий.

Ключевые слова: цепь поставок, аддитивные технологии, промышленное производство, логистическая система.

На сегодняшний день все развитые и крупнейшие развивающиеся страны мира в полной мере осознают наступление нового (шестого) технологического уклада. Начало перехода к новому технологическому укладу неизменно характеризуется высоким темпом роста емкости рынков ключевых производственных технологий, нередко носящим экспоненциальный характер. Следуя данному тезису, в рамках шестого технологического уклада могут быть выделены следующие рынки:

1. Рынок интеллектуальных систем автоматизации процесса проектирования.

2. Рынок гибких производственных комплексов.

3. Рынок промышленных аддитивных установок, гибридных аддитивных установок и средств печати.

4. Рынок адаптивной робототехники и средств межмашинного взаимодействия.

5. Рынок индустриального интернета.

6. Рынок промышленных дронов.

Совокупность данных рынков можно назвать индустрией передовых производственных технологий. В 2016 году объем данной индустрии составлял всего 66,7 млрд. \$, в то время как к 2022 году прогнозируется его рост до 152, 3 млрд. $\$$ [7]. Средний темп роста при этом должен составить $14,7 \%$ в год, что значительно выше, чем у рынков «традиционных» технологий. Последствия данного роста носят дуалистичный характер. С одной стороны, увеличение емкости рынка приводит к повышению уровня конкуренции, следствием чего становится совершенствование потребительских свойств товаров и снижение себестоимости производства, в первую очередь за счет достижения эффекта от масштаба. Потребность в высококвалифицированных специалистах на данных рынках также динамично увеличивается, что приводит к завышению цены труда данных специалистов. С другой стороны, развитие данных технологий неизбежно приводит к изменениям производственных процессов, за счет изменения свойств основных производственных фондов, а также свойств сырья и материалов. В связи с этим, значительные трансформации претерпевают цепи поставок. Данные трансформации обусловливают как изменение географии поставок, так и изменение свойств транспортируемых грузов. 
Наиболее значительные изменения цепи поставок претерпевают в связи с развитием рынка аддитивных технологий. Под аддитивными технологиями понимается комплекс технологий, предполагающих изготовление изделия на основе цифровой модели методом послойного добавления материала [1]. Развитие данных технологий может оказать значительное влияние на мировые промышленные рынки. В статье [9] авторы утверждают, что аддитивные технологии могут выступать источником новой промышленной революции. В качестве одной из отличительных особенностей данного комплекса технологий выделяется возможность производства изделия уникальной геометрии без применения специализированного инструмента и в рамках одной производственной операции. Данные свойства могут привести к снижению трудоемкости производства и, следовательно, производственной себестоимости. В рамках статьи [6] авторы декларируют возможность изменения географии мировой промышленности, считая, что существующая на данный момент привязанность промышленных предприятий к центрам добычи сырья может быть в значительной мере ослаблена вследствие развития аддитивных технологий. В первую очередь это обусловлено высоким уровнем возвратности сырья и его относительно однородной структурой. Авторы данной статьи в полной мере разделяют указанную точку зрения и предполагают, что именно данные свойства аддитивного производства могут привести к изменениям в цепях поставок. В статье [5] также рассматриваются аддитивные технологии как источник промышленной революции. В качестве основных преимуществ аддитивных технологий выделяется снижение постоянных издержек предприятия. В статье [8] авторы выделяют в качестве основного преимущества аддитивных технологий значительное приращение возможностей конструирования сложных форм, так как процесс производства изделий любой формы незначительно отличается с точки зрения производственной себестоимости, в первую очередь за счет отсутствия необходимости в специализированной оснастке и/или дополнительной работе технолога, про- граммиста и оператора станка. Таким образом, можно заключить, что аддитивные технологии могут в значительной мере повлиять на мировую промышленность, замещая традиционные технологии автоматизированной механической обработки материалов (CNC технологии). К ключевым предпосылкам данного процесса можно отнести:

1. Отсутствие невозвратных отходов. Все отходы могут быть вновь переработаны и направлены в производство. Это обеспечивается за счет того, что используемый материал однороден.

2. Отсутствие невозвратного брака. Весь брак также, как и производственные отходы, может быть переработан и заново направлен в производство.

3. Снижение энергоемкости производимой продукции. Аддитивные установки требуют значительно меньше энергии, нежели классическое CNC оборудование.

4. Универсальность средств производства. Принцип послойной печати позволяет производить изделия практически любой сложности [2].

5. Снижение трудоемкости производства. Использование аддитивного оборудования позволяет значительно снизить потребность в персонале.

Данные предпосылки позволяют утверждать, что традиционное промышленное производство постигнет неизбежная трансформация под воздействием развития аддитивных технологий. Данная трансформация также неизбежно приведет к содержательным и структурным изменениям глобальных цепей поставок. В первую очередь необходимо рассмотреть возможные содержательные изменения глобальной цепи поставок. Традиционная модель данной цепи представлена на рисунке 1.

С содержательной точки зрения основные изменения традиционной модели цепи поставок в процессе развития аддитивных технологий сосредоточатся на этапах $1-$ «Поставщик» и 2 - «Производитель». Рассмотрим данные этапы последовательно. В первую очередь, надо отметить изменение структуры поставляемых оборотных производственных фондов (сырья)
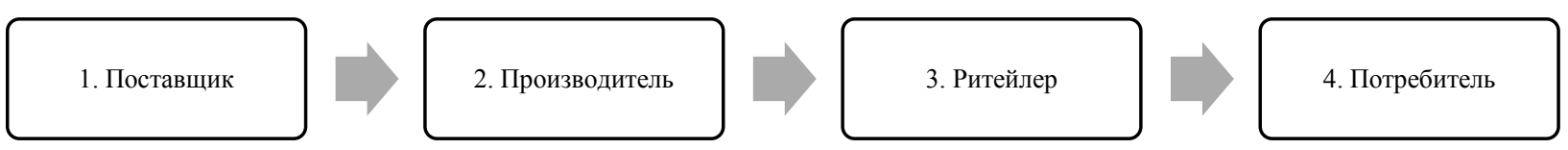

Рuc. 1. Традиционная модель цепи поставок $[3,4]$ 
конечным производителям. Как уже отмечалось выше, применение промышленных аддитивных установок подразумевает однородность используемого сырья. Следовательно, отсутствует необходимость в габаритной дифференциации процесса поставок. Физические и химические свойства данного сырья, как правило, не подразумевают организации особых условий транспортировки, что значительно снижает барьеры входа на данный рынок малых транспортных компаний. Данные свойства также распространяются на процесс хранения. Подобное сырье практически не чувствительно к перепадам температуры, влажности и наличию каких-либо примесей в атмосфере. Хранение в специализированных емкостях, позволяющих максимизировать полезный объем складских помещений, дает возможность увеличить запасы сырья без расширения склада.

Однако, на данный момент рынок производителей материалов для 3D печати достаточно ограничен. Это обусловлено как недостаточной емкостью рынка аддитивных технологий в целом, так и высокими техническими и экологическими требованиями к процессу производства. На сегодняшний день, производство многих видов материалов для 3D печати ограничено несколькими малыми предприятиями, что не позволяет обеспечить конкурентные цены на рынке. Также необходимо отметить, что многие производители аддитивных установок закладывают в них возможность эффективной печати исключительно из материала определенного производителя, что также негативно сказывается на конкурентной среде. Однако, несмотря на выделенные недостатки, следует прогнозировать увеличение конкуренции на рынке логистических услуг, как следствие увеличения емкости рынка аддитивных технологий. При этом надо отметить, что процесс транспортировки самих аддитивных установок практически не отличается от процесса транспортировки машин и оборудования для механической обработки материалов. Значительно более значимые изменения стоит прогнозировать на этапе производства. В первую очередь следует в очередной раз отметить значительное снижение доли затрат на оплату труда производственного персонала в формировании себестоимости производства. Аддитивные технологии позволяют в значительной мере автоматизировать процесс производства. В действительности, незаменимым остаётся исклю- чительно персонал, обеспечивающий процесс конструирования продукции. Снижение трудоемкости процесса взаимодействия с основными производственными фондами дает возможность расширения функционала существующего производственного персонала. Также необходимо отметить возможность упрощения систем организации и управления складом. Данная возможность обеспечивается обозначенной ранее однородностью сырья и материалов. Более того, практически полная возвратность отходов и производственного брака позволит практически отказаться от систем утилизации. Совокупность вышеперечисленного позволяет предполагать возможность снижения потребности в складских площадях, и возможность организации автоматизированных систем хранения и транспортировки материалов. Создание данных систем может стать одним из основных направления развития складской логистики в ближайшие годы. Процесс транспортировки готовой продукции до ритейлера, и далее до потребителя практически не претерпевает содержательных изменений в рамках традиционной цепи поставок. Единственное значимое изменение, являющиеся непосредственным следствием развития рынков аддитивных технологий, это возможность создания уникальной тары и упаковки, позволяющей как обезопасить объект в процессе транспортировки, так и создать дополнительную потребительскую ценность.

Выделенные содержательные изменения традиционной цепи поставок безусловно являются значительными, и во многом трансформируют существующие логистические системы, подходы к транспортировке и хранению и т.д. Однако, развитие рынков аддитивных технологий раскрывает возможность не только содержательных, но и структурных изменений традиционной цепи поставок. В статье [3] автором выделяется две возможные формы структурной трансформации традиционной цепи поставок: цепь поставок «3D магазин» и цепь поставок «Домашняя печать». Рассмотрим данные формы последовательно. На рисунке 2 представлена модель цепи поставок «3D магазин».

В рамках данной модели цепи поставок предполагается полное исключение крупных промышленных производителей. Предполагается разделения конструкторского и производственного функционала предприятия [4]. При этом потребитель не ощущает непосредствен- 


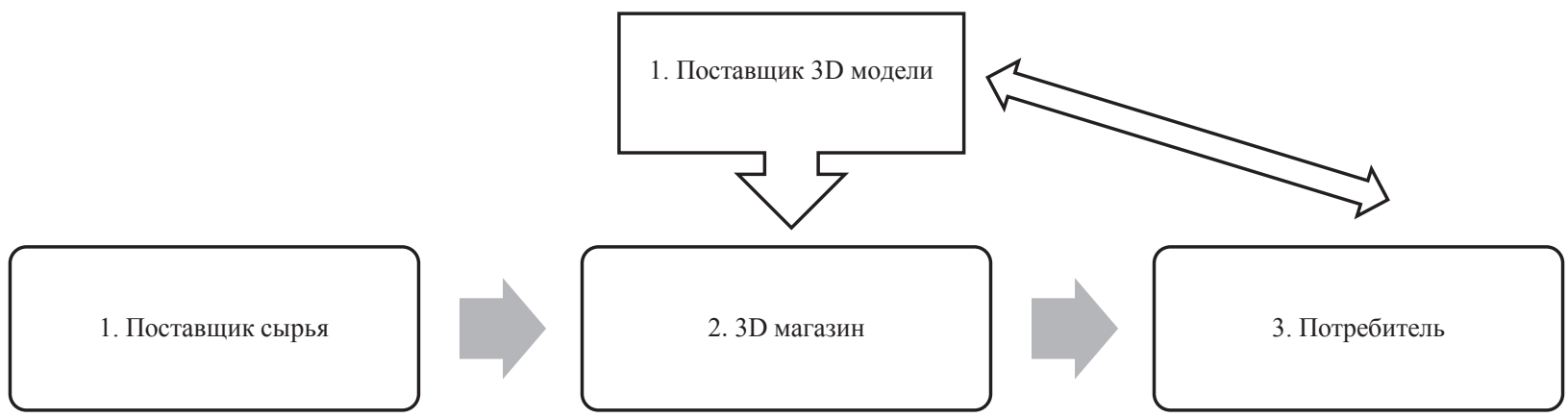

Puc. 2. Цепь поставок «3D магазин»
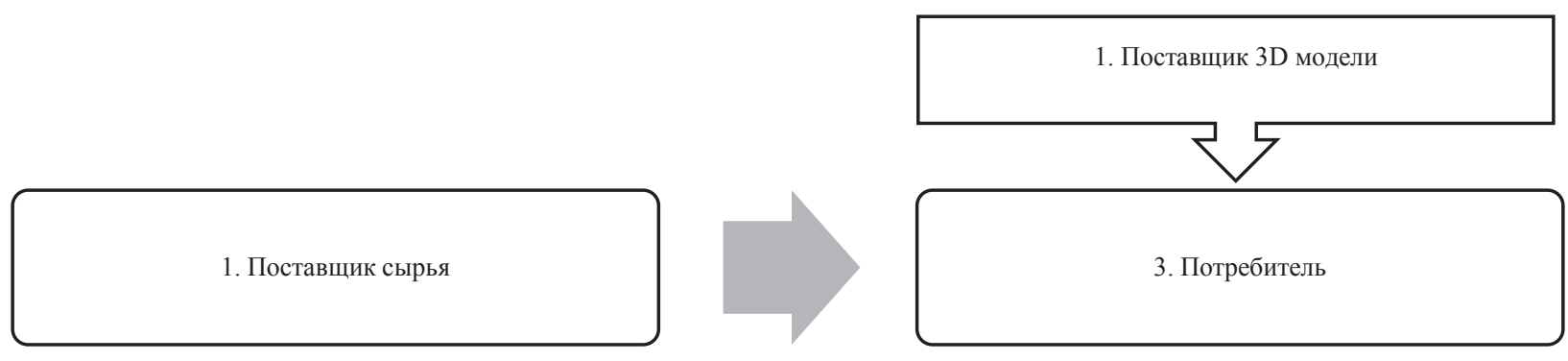

Рис.3. Цепь поставок «Домашняя печать»

ной разницы в свойствах конечного продукта. Появление подобной цепи поставок становится возможным исключительно благодаря значительному снижению трудоемкости процесса производства по сравнению с классическими способами механической обработки. При этом поставщиком самой 3D модели может выступать как сам потребитель, так и внешнее конструкторское бюро. В рамках данной модели цепи поставок возможна организация процесса удаленной коммуникации, при которой поставщик 3D модели может находиться на любом удалении от аддитивной установки. Данная модель в первую очередь отличается своей универсальностью и масштабируемостью. Однако, система взаимодействия 3D магазина и потребителя, в части транспортировки готовой продукции, не отличается от традиционной. Завершающая модель структурной трансформации представлена на рисунке 3.

В рамках данной модели цепи поставок предполагается полное исключение производителя. В данном случае процесс производства реализуется самим потребителем. Развитие данной модели цепи поставок предполагает значительное сокращение промышленного сектора, при одновременном развитии логистических сервисов. Необходимость организации поставок сырья и материалов для печати малыми и не постоянными партиями неизбежно приведет к появлению отдельного рынка логистических посредников, способных работать непосредственно с потребителями рынка В2С. Вероятно, данный рынок может быть сформирован в первую очередь за счет транспортных компаний, спрос на услуги которых со стороны промышленного сектора сократится в связи с развитием данной модели цепи поставок.

\section{Библиографический список}

1. Конников E.А. Опционный подход к оценке устойчивости развития промышленных предприятий в процессе перехода на передовые производственные технологии (на примере аддитивных технологий) // Конкурентоспособность в глобальном мире: экономика, наука, технологии. 2017. № 8-5 (55). С. 37-45.

2. Курочкина А.Ю., Голубцов С.Б., Погребова О.А. Интернет-маркетинг: Учебное пособие. Санкт-Петербург. 2016. 
3. Москвитина Е.И., Толкачев С.А. Формирование новых контуров управления глобальными цепочками стоимости под воздействием аддитивных технологий // Управленческие науки. 2016. Т. 6. № 4. С. 85-94.

4. Мясникова Л.А Логистика аддитивных производств // Вестник факультета управления СПбГЭУ. 2017. № 1-1. C. 419-422.

5. Cozmei C., Caloian F. Additive manufacturing flickering at the beginning of existence // Procedia Economics and Finance. 2012. № 3. P. 457-462.

6. Gress D.R., Kalafsky R.V. Geographies of production in 3D: Theoretical and research implications stemming from additive manufacturing // Geoforum. 2015. № 60. P. 43-52.

7. Konnikov E.A., Pogrebova O.A., Maskova Yu.R., Glukhov V.V. Real options valuation of additive production // B сборнике: Reliability, Infocom Technologies and Optimization (Trends and Future Directions) 6th International Conference ICRITO. 2017. P. . 557-563.

8. Mellor S., Hao L., Zhang D. Additive manufacturing: A framework for implementation // International Journal of Production Economics. 2014. No 149. P. 194-201.

9. Weller C., Kleer R., Piller F. T. Economic implications of 3D printing: Market structure models in light of additive manufacturing revisited // International Journal of Production Economics. 2015. No 164.P. 43-56.

Поступила в редакцию 09.09.2018 